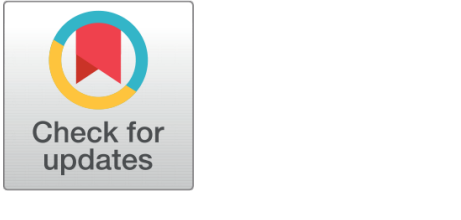

open ACCESS

Received: 19.04.2021

Accepted: 15.07.2021

Published: 09.08.2021

Citation: Bharathi T, Antony Vinoth S, Leo S (2021) Fuzzy Radio Reciprocal L-Labeling on Certain Chemical Graphs. Indian Journal of Science and Technology 14(27): 2284-2292. https://doi.org/ 10.17485/IJST/v14i27.664

* Corresponding author. annyvino@gmail.com

Funding: None

Competing Interests: None

Copyright: () 2021 Bharathi et al. This is an open access article distributed under the terms of the Creative Commons Attribution License, which permits unrestricted use, distribution, and reproduction in any medium, provided the original author and source are credited.

Published By Indian Society for Education and Environment (iSee)

ISSN

Print: 0974-6846

Electronic: 0974-5645

\section{Fuzzy Radio Reciprocal L-Labeling on Certain Chemical Graphs}

\author{
T Bharathi ${ }^{1}$, S Antony Vinoth ${ }^{2 *}$, S Leo $^{2}$ \\ 1 Assistant Professor, Department of Mathematics, Loyola College, University of Madras, \\ Chennai, Tamil Nadu, India \\ 2 Research Scholar, Department of Mathematics, Loyola College, University of Madras, \\ Chennai, Tamil Nadu, India
}

\section{Abstract}

Objectives: The objective of this study is to reduce the interference of the signals between Wi-Fi devices where the fuzzy values of the frequencies are from the closed interval $[0,1]$. Method: A new methodology is introduced to reduce the interference of the signals between Wi-Fi devices that is fuzzy radio reciprocal L-labeling. Findings: Here the general formula of fuzzy radio reciprocal L-labeling has been newly introduced to apply this concept in chemical graphs. Further results and discussions are also proved in this connection using the fuzzy chemical graph structure, where the distance and the frequencies between the routers of Wi-Fi connections are assigned fuzzy weights based on fuzzy radio reciprocal L-labeling, so that interference can be reduced and the signal strength is optimized.

Keywords: Nano sheet; Sirpinski gasket graph ( S n ); Sirpinski like graph (S (n; 4)); $\gamma(\mathrm{s})$ weight of lines; $\tau$ (a) weight of points

\section{Introduction}

Graph theory is related with numerous divisions of mathematics like group theory, matrix theory, numerical analysis, probability and topology. It has also applications in other scientific subjects such as physics, chemistry, communication science, computer technology, electrical engineering, and so on. Graph theory is also associated with the field of chemical graph theory.

There are many reasons for the increasing popularity of graph theory in chemistry. Chemical graph theory is a subdivision of mathematical chemistry in which points denote atoms and lines denote chemical bonds in the underlying chemical structure. Graph theory offers simple rules by which chemists obtain qualitative predictions about the structure and reactivity of various compounds. Graph theory is used as a basis for the representation, classification, and categorization of a very huge chemical system and carbon Nano structures. In the field of chemistry, graph theory is more useful theoretical tool. The study of fuzzy radio reciprocal L- labeling is applicable to chemical graphs like V-Phenylenic Nanosheets, Sirpinski graphs. In fuzzy radio reciprocal L- labeling of a chemical graph, weight of points and lines are assigned from the interval $(0,1)$. $\mathrm{L}$ is the length of any two pair of points and it has the main role to assign weights to every other of the points to reach the goal. 
The frequency of assignment problem is to assign a frequency to each Wi-Fi router from the interval $(0,1)$. Even if each Wi-Fi router's frequency is very close, there is no interference among the routers. So it gives better signal to use internet connections, like Hot spots etc. The generalization formula of fuzzy radio reciprocal L-labeling number is derived for Nano sheet and two types of Sirpinski graphs.

Notations: Edges- lines, Vertices-points, A- Set of points in fuzzy graph, S- Set of lines in fuzzy graph, Weight- Membership value of fuzzy graph, $\gamma(s)$ - Weight of line, $\tau(a)$ - Weight of points, $\mathrm{k}$ - Depends on digits of natural number, L- Number of lines in between any two points which is the distance of points, $\delta\left(a_{i}, a_{j}\right), \mu$-distance of two points $a_{i}$ and $a_{j}$, Diam-Diameter of fuzzy graph.

\section{Literature review}

Hale (1980) formulated graph vertex coloring problem based on the frequency assignment problem where a frequency is assigned to each radio transmitter ${ }^{(1)}$. Griggs and Yeh (1992) proposed that the $\mathrm{L}(2,1)$-labeling of a simple graph $\mathrm{G}$ is a function $\mathrm{g}$ from the vertex set $\mathrm{V}(\mathrm{G})$ into the nonnegative integers such that if the distance of any two vertices $a$ and $b$ is 1 , then the difference of cardinality of $\mathrm{g}(\mathrm{a})$ and $\mathrm{g}(\mathrm{b})$ is greater than or equal to 2 and if the distance of any two vertices $\mathrm{a}$ and $\mathrm{b}$ is 2 , then the difference of cardinality of $\mathrm{g}(\mathrm{a})$ and $\mathrm{g}(\mathrm{b})$ is greater than or equal to 1 .The span off is the absolute difference between the largest assigned label and the smallest assigned label of $\mathrm{g}^{(2)}$. Heuvel et al. (1998) discussed a model for the way in which interference is avoided in real radio systems. Each pair of vertices has, depending on their separation, a constraint on the difference between the labels that can be assigned. They find labeling of minimum span, for given a graph and a set of constraints. Their focus is on the infinite triangular lattice, infinite square lattice, and infinite line lattice, and optimal labelings for up to three levels of constraints and they highlight how accepted practice can lead to suboptimal channel assignments ${ }^{(3)}$. Shao \& Zhang (2008) derive upper bound of Total graphs and conclude that Griggs and Yeh's conjecture is true for the total graph in some cases. They discussed about an i-stable set (i.e.) the distance between any two vertices in a subset $\mathrm{A}$ of vertices is greater than $\mathrm{i}$. A 1-stable set is a usual independent set. A maximal 2-stable subset A of a set B is a 2-stable subset of B such that A is not a proper subset of any 2-stable subset of $\mathrm{A}^{(4)}$.

Gani \& Radha (2009) found the degree of vertices in union of two fuzzy graphs, sum of two fuzzy graphs and Cartesian product of two fuzzy graphs ${ }^{(5)}$. Panigrahi (2009) explained the frequency assignment problem from the discovery that transmitters, received the same or closely related frequencies, had interferences with one another. Nearly three decade back this problem has been modelled as a graph labelling problem. This labelling has several variations depending upon the type of assignment of frequencies to transmitters. They discussed about some of them and gave a detailed survey on the most recent frequency assignment problem called the radio k-colouring of graphs ${ }^{(6)}$. Massadeh (2011) proposed complete, regular and complement fuzzy graph. They studied some properties of self-complementary, regular, total regular fuzzy graph. The relation between the product, complete and complement of fuzzy graphs are proved ${ }^{(7)}$. Nagoorgani \& Subahashini (2014) introduced the concept fuzzy labeling and fuzzy magic labeling graph and defined fuzzy magic labeling for path, cycle, and star graph. They proved that every fuzzy magic graph is a fuzzy labeling graph, but the converse is not true and showed that the removal of a fuzzy bridge from a fuzzy magic cycle with odd nodes reduces the strength of a fuzzy magic cycle. Some properties related to fuzzy bridge are discussed ${ }^{(8)}$. Nagoorgani \& Subahashini (2014) extended the fuzzy labeling concept to fuzzy tree. Different properties of fuzzy labeling trees are discussed and also bipartite fuzzy labeling graph is defined and properties of fuzzy labeling are discussed. It contains an algorithm for finding the fuzzy spanning subgraph for a fuzzy labeling tree and finding the maximum spanning, strong arcs and fuzzy bridges of any fuzzy labeling graph ${ }^{(9)}$. Tom \& sunith (2015) proved strong sum distance in fuzzy graphs is metric and the distance of a fuzzy graph is greater than or equal to its radius of fuzzy graph and less than or equal radius with multiple 2 of fuzzy graph ${ }^{(10)}$. Nazeer et al. (2016) discussed the radio labeling of Generalized Caterpillar graphs $\mathrm{C}(\mathrm{m}, 1) \mathrm{Pn}$, which is obtained from Pn by attaching $\mathrm{m}$ vertices of degree two to each vertex of degree two of Pn. They found a generalized result for radio labelling number in Generalized Caterpillar graphs ${ }^{(11)}$. Mahapatra et al. (2019) introduced radio fuzzy graph. They noted that the edge membership value depends on the chosen value of distance and it decreases as the edge membership value will increase. As a result, the set-up cost becomes high. In addition, need more space is needed for such a set-up. Thus, cost and air distance are counter proportional to each other. There are two major targets investigated to assign numbers to the vertices, the span should be minimum (i.e.) the difference of lowest and highest labels must be minimum and to assign a minimum number of basic colors is assigned. Both may or may not be satisfied in a single assignment ${ }^{(12)}$. Stephen John et al (2020) obtained a general formula of radio labeling of complete related graphs and also they compared the radio labelling number of path and cycle. They proved the result that if, If the order of path and number of vertices are same, then radio labelling number of path and cycle are same. The results of radio labelling number of centipede graph, banana tree, ladder graph, fire cracker graph are attained ${ }^{(13)}$. Uma et al. (2020) obtained radio labeling of some corona graphs and its radio number. They considered only simple, finite, connected and undirected graphs. They found the result of radio number of corona graphs 
$3 n+8^{(14)}$. Angel Chavez et al. (2021) discussed optimal radio $k$-labelling of a tree. A tree radio-k-number is equal to the lower bound of a k-lower bound tree and establish properties of k-lower bound trees, and applied these properties to obtain shorter proofs and generalizations of some known results. They investigate the minimum integer $\mathrm{k}$ such that a given tree $\mathrm{T}$ is a $\mathrm{k}-\mathrm{lower}$ bound tree and defined the joined union operation on trees, where trees are composed by merging a vertex from each tree into a single vertex ${ }^{(15)}$.

\section{Methodology}

In general, the focus on radio labeling is to avoid interference between any two radio stations. If the distance between any two radio stations is close, then the interference is unavoidable. In 2005, Chartrand et al. introduced the concept of radio labeling and put the level of interference at largest possible-the diameter of graph. For any graph, points are labeled by the positive integers and then the sum of the difference between of any two labeled points $\mathrm{a}$ and $\mathrm{b}$ and distance between $\mathrm{a}$ and $\mathrm{b}$ is greater than or equal to diameter of graph. Even if distance is close, then difference between the values assigned to the concerned radio channels is high. In radio labeling distance depends on the number of minimum lines between any two radio stations ${ }^{(16)}$. In this study, For any fuzzy graph and fuzzy radio reciprocal L- labeling, points and lines are assigned fuzzy weights lying between 0 and 1 . Here the distance is obtained through the concept of $\mu$-distance which depends on the weights of line or lines between any two points. By the concept of $\mu$ - distance, distance between any two points lie $[1, \infty)$ and diameter of fuzzy graph lie $[1, \infty)$. In fuzzy radio reciprocal L- labeling even if the distance is close, the interference between any two Wi-Fi routers can be avoided and thus connectivity of Wi-Fi routers is very smooth.

\section{Discussion}

In radio channel assignment problems, the channel frequency difference between two channels is $0.2 \mathrm{MHz}$. For broadcasts in air, the available bandwidth is determined by the allocated frequency band. For example, AM radio signals must be in the 535$1605 \mathrm{kHz}$ band. Therefore, there are 107 possible carrier frequencies that may be assigned, at $10 \mathrm{kHz}$ intervals. Likewise FM radio stations are at $0.2 \mathrm{MHz}$ intervals in the range of $88-108 \mathrm{MHz}$ allowing 100 stations in a local area ${ }^{(16)}$. But based on fuzzy radio reciprocal L- labelling, the difference between any two $\mathrm{Wi}$-Fi routers is taken as $0.01 \mathrm{GHz}, 0.02 \mathrm{GHz}, 0.03 \mathrm{GHz}$ etc., $\mathrm{Here}$ $\mathrm{Wi}-\mathrm{Fi}$ routers are fixed depending on usage and the distance between them. The difference between any two Wi-Fi routers vary depending on decimals lying between 0 and 1.

\section{Basic preliminaries}

Definition 4.1. A fuzzy graph $F G=(A, \gamma, \tau)$ is a pair of function $\tau: A \rightarrow[0,1]$ and $\gamma: A \times A \rightarrow[0,1]$, where $\gamma\left(a_{i}, a_{j}\right) \leq \tau\left(a_{i}\right) \wedge$ $\tau\left(a_{j}\right)$. A path $\mathrm{P}$ in a fuzzy graph is a sequence of distinct nodes $a_{i}=a_{0}, a_{1} \ldots a_{n}=a_{j}$ such that $\gamma\left(a_{x-1}, a_{x}\right)>0,1 \leq x \leq n, n \geq 0$ is called the length of $\mathrm{P}$. The consecutive pairs of $\gamma\left(a_{x-1}, a_{x}\right)$ are called the arcs of the path. The $\mu$-distance is the smallest $\mu$ -length of any $a_{i}-a_{j}$ path $\mathrm{P}$, where $\mu$ - length of a path is the sum of the reciprocal of the line weights in $\mathrm{P}$ and the diameter is maximum of $\mu$-distance of $\mathrm{FG}^{(17,18)}$.

Definition 4.2. A graph $G=(A, \gamma, \tau)$ is said to be a fuzzy labeling graph if $\tau: A \rightarrow[0,1]$ and $\gamma: S \rightarrow[0,1]$ is bijective such that weight of lines and points are specified and $\gamma\left(a_{i}, a_{j}\right)<\tau\left(a_{i}\right) \wedge \tau\left(a_{j}\right)^{(19)}$.

Definition 4.3. V-Phenylenic nanotubes $\operatorname{VPH}(\mathrm{m}, \mathrm{n})$ are molecular graphs which are given by $C_{6}, C_{4}, C_{8}$, where m indicates the number of rows and $n$ indicates the number of columns. In the V-Phenylenic Nano sheet, $m$ indicates the number of hexagons in every column and $n$ indicates the number of hexagons in every row ${ }^{(20)}$.

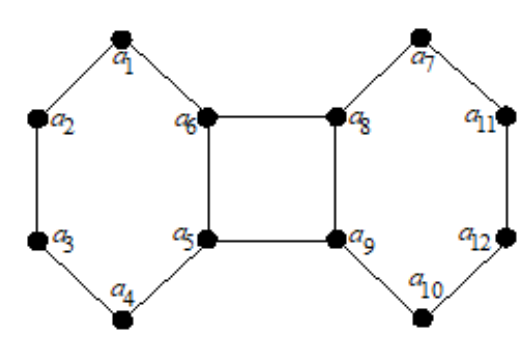

Fig 1. V-Phenylenic nanosheet 
Definition 4.4. The Sirpinski gasket graph $\left(S_{n}\right), n \geq 1$, is obtained from $S(n, 3)$ by contracting all the edges of $S(n, 3)$ that lie in no triangle ${ }^{(21)}$.

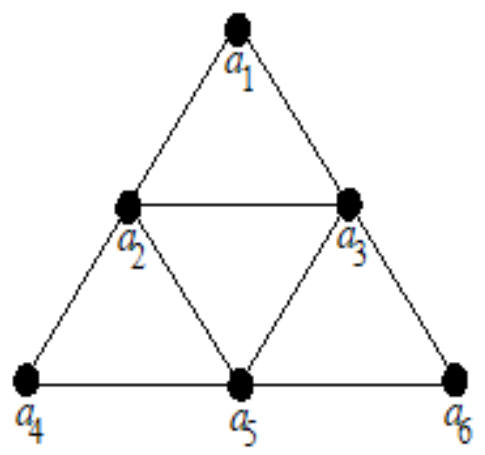

Fig 2. Sirpinski gasket graph

Definition 4.5. The Sirpinski graph $\mathrm{S}(\mathrm{n}, \mathrm{k})$, is defined for $n \geq 1$ and $k \geq 1$ as follows: The point set of $\mathrm{S}(\mathrm{n}, \mathrm{k})$ is made up of all $\mathrm{n}$-tuples of integers $1,2,3, \ldots, \mathrm{k}$ that is, $\operatorname{VS}(n, k)=\{1,2,3 \ldots\}^{n}$. Two different points $\left(a=a_{1}, a_{2}, a_{3} \ldots a_{n}\right)$ and $\left(b=b_{1}, b_{2}, b_{3} \ldots b_{n}\right)$ are adjacent if only if there exists an $h \in\{1,2 \ldots n\}$ such that

1. $a_{t}=b_{t}$ for $t=1,2 \ldots h-1$

2. $a_{h} \neq b_{h}$; and

3. $a_{t}=b_{t}$ and $b_{t}=a_{h}$ for $\mathrm{i} t=h+1, \ldots n$.we write $\left(a_{1} a_{2} a_{3} \ldots a_{n}\right)$ or even shorter $\left(a_{1}, a_{2}, a_{3} \ldots a_{n}\right)$ Consider the case when $\mathrm{k}$ $=4$ then Sirpinski like graph denoted $S(n, 4)^{(21)}$

\section{Results}

A fuzzy graph $F G=(A, \gamma, \tau)$ is said to be a fuzzy radio reciprocal L- labeling if $\tau: A \rightarrow[0,1]$ and $\gamma: S \rightarrow[0,1]$ is bijective then following condition is satisfied,

$$
\delta\left(a_{i}, a_{j}\right)+L \times\left(\frac{1}{\left|\tau\left(a_{i}\right)-\tau\left(a_{j}\right)\right|}\right) \geq \operatorname{diam}(F G)
$$

where $\delta\left(a_{i}, a_{j}\right)$ is the $\mu$-distance of the fuzzy graph, diam(FG) is the maximum of the $\mu$-distance of every pair of points, $\mathrm{L}$ is the number of lines connecting corresponding points of graph FG (i,e.) $\mathrm{L}=\left|P_{s}\right|$ where $s$ is the number of lines in the path $\mathrm{P}$ connecting the corresponding points of the graph FG. Fuzzy radio reciprocal L- labeling is denoted by $F R_{L L}^{-1}(22)$. The highweights of the points is taken minimum as possible. This is known as fuzzy radio reciprocal $L$ - labeling number denoted by $F R_{L L}^{-1}(m)$.

\subsection{Fuzzy radio reciprocal L-labeling on Nano sheet}

V-Phenylenic nanotubes VPH(m, n) is called fuzzy V-Phenylenic N-tubes FVPH(m, n), $F V P H(m, n)=(A, \gamma, \tau)$ of a nonempty set together with a pair of function $\tau: A \rightarrow[0,1]$ and $\gamma: S \rightarrow[0,1]$ if $\gamma\left(a_{i}, a_{j}\right)=\tau\left(a_{i}\right) \wedge \tau\left(a_{j}\right)$ for all $a_{i}, a_{j} \in A$ denoted by $\operatorname{FVPH}(\mathrm{m}, \mathrm{n})$.

Lemma 5.1.1: For any n- dimensional fuzzy chemical graph, the weights of points and lines lie between $\left(\frac{5}{10^{k}}, \frac{9}{10^{k}}\right)$.

Proof: Let FCHG be any n- dimensional chemical graph with points $A=\left\{a_{1}, a_{2}, a_{3} \ldots a_{n}\right\}$ and lines $S=\left\{s_{1}, s_{2}, s_{3} \ldots s_{n}\right\}$. Assign weight to every point and line. For any two points $\left(a_{i}, a_{j}\right)$ there is one and only one line between $\left(a_{i}, a_{j}\right)$. The difference between weights of lines $a_{i}$ and $a_{j}$ is less than or equal to $\frac{3}{10^{k}}$. When this is maximum, then the reciprocal of the difference between weights of $\left(a_{i}, a_{j}\right)$ is minimum. Let us take any other two points $a_{b}$ and $a_{c}$, where $a_{b}, a_{c} \in A$. When $\mu$ - distance of $a_{b}$ and $a_{c}$ is maximum and it is also the diameter of fuzzy chemical graph, then.

$$
\delta\left(a_{i}, a_{j}\right)+L \times\left(\frac{1}{\left|\tau\left(a_{i}\right)-\tau\left(a_{j}\right)\right|}\right) \leq \operatorname{diam}(F G)
$$


Therefore the weight of points and lines lie between $\left(\frac{5}{10^{k}}, \frac{9}{10^{k}}\right)$ as much possible to satisfies

$$
\delta\left(a_{i}, a_{j}\right)+L \times\left(\frac{1}{\left|\tau\left(a_{i}\right)-\tau\left(a_{j}\right)\right|}\right) \geq \operatorname{diam}(F G)
$$

Lemma 5.1.2: For any n- dimensional fuzzy V-Phenylenic Nano sheet, $\operatorname{diam}(F V P H(m, n))<\left|n P_{b}\right|$, where $\left|n P_{b}\right|$ is the number of lines in the boundary of Nano sheet.

Proof: Let us take any n- dimensional fuzzy V-Phenylenic Nano sheet, assign the weight to the lines which are always less than the corresponding weights of the points. When weights are allotted to the lines in $\mathrm{FVPH}(\mathrm{m}, \mathrm{n})$ then the reciprocal the weight of any weight of the lines must be 1 or $1 . x x \ldots x$ where $\mathrm{x}$ varies from 1 to 9 .

When $\mathrm{m} \geq \mathrm{n}$

The sum of the number of lines on the boundary of $R_{m, 1}$ and the number of lines on the boundary of $C_{m, n}$ are less than $\operatorname{diam}(F V P H(m, n))$.

When $\mathrm{m}<\mathrm{n}$

The sum of the number of lines on the boundary of $C_{1, n}$ and the number of lines on the boundary of $R_{m, n}$ are less than $\operatorname{diam}(F V P H(m, n))$. By lemma 5.1.1. When there is one line between $a_{i}$ and $a_{j}$ as well as the values of $\left(a_{i}, a_{j}\right)$ lie between $[1$ ,1.xx...x) then the $\operatorname{diam}(V P H(m, n))<\left|n P_{b}\right|$.

Lemma 5.1.3: In a $n$ - dimensional fuzzy Sirpinski graph $(n, k)$ the diameter is less than the sum of the lines on the boundary which is a cycle and $\mathrm{k}=3,4$.

Proof: In an n-dimensional Sirpinski graph $(\mathrm{n}, \mathrm{k}), \delta\left(a_{i}, a_{j}\right)$ refers to the distance between any two points. The distance between any points is determined by the weight of the lines and the minimum $\mu$ - length of the corresponding points. The maximum among the distances between any pair of points is the diameter of fuzzy Sirpinski graph by lemma 5.1.2. By lemma 5.1 .1 distance between any pair of points is $1 \leq \delta\left(a_{i}, a_{j}\right)<2$. According to this range the diameter of the chemical graph must be less than the number of lines in the cycle on the boundary of the fuzzy Sirpinski graph.

Theorem 5.1.4: If the diameter of $\operatorname{FVPH}(m, n)$ is less than the sum of the number of lines on the boundary of $\mathrm{FVPH}(\mathrm{m}, \mathrm{n})$ , then $\delta\left(a_{i}, a_{j}\right)+L \times\left(\frac{1}{\left|\tau\left(a_{i}\right)-\tau\left(a_{j}\right)\right|}\right) \geq \operatorname{diam}(F G)$.

Proof: Let VPH(m, n) be the $\mathrm{n}$-dimensional Nano sheet with points $A=\left\{a_{1}, a_{2}, a_{3} \ldots a_{t}\right\}$ and lines $S=\left\{s_{1}, s_{2}, s_{3} \ldots s_{t}\right\}$. Assign weights to lines by

$$
\gamma\left(s_{p}\right)=\left(\frac{\left(T \times 10^{q}\right)}{10^{k}}+\frac{p}{10^{k}}\right), R>T, \mathrm{~T}=4,5,6,7,8,9, \mathrm{p}=0,1,2,3 \ldots, q=k+1 .
$$

Proceeding similar way assign weights to points by

$\tau\left(a_{t}\right)=\left(\frac{\left(R \times 10^{q}\right)}{10^{k}}+\frac{t}{10^{k}}\right), \mathrm{R}=5,6,7,8,9, \mathrm{t}=1,2,3 \ldots, q=k+1$.

From $\tau\left(a_{t}\right)$ and $\gamma\left(s_{p}\right)$ by lemma 5.1 .1 it satisfies

$$
\gamma\left(a_{i}, a_{j}\right)<\min \left(\tau\left(a_{i}\right), \tau\left(a_{j}\right)\right), a_{i}, a_{j} \in A
$$

To prove: $\operatorname{FVPH}(\mathrm{m}, \mathrm{n})$ satisfies $\delta\left(a_{i}, a_{j}\right)+L \times\left(\frac{1}{\left|\tau\left(a_{i}\right)-\tau\left(a_{j}\right)\right|}\right) \geq \operatorname{diam}(F G)$.

Let $\delta\left(a_{i}, a_{j}\right)$ be the distance between any two points then theorem is proved as follows:

When $\mathrm{m} \geq \mathrm{n}$ the following cases hold true. Assign weights of points row-wise,

Case (i): When $\left|\tau\left(a_{i}\right)-\tau\left(a_{j}\right)\right|=\frac{5}{10^{k}}, \mathrm{i}=1,2,5 \ldots$ and there is only one line between the points $a_{i}$ and $a_{j}$, i.e., $L=\left|n P_{d}\right|=1$, then the reciprocal of $\left|\tau\left(a_{i}\right)-\tau\left(a_{j}\right)\right|$ is more or less the maximum and $F R_{L L}^{-1}$ is satisfied.

Case (ii): Assign weights to points row-wise $\left(R_{11}, R_{21} \ldots R_{m 1}, R_{m 2}, \ldots R_{m n}\right)$ when $\left|\tau\left(a_{i}\right)-\tau\left(a_{j}\right)\right|=\frac{6 m-5}{10^{k}}$, m=2, 3 and there is only one line between the points $a_{i}$ and $a_{j}$ i.e., $L=\left|n P_{b}\right|=1$, then the Reciprocal of $\left|\tau\left(a_{i}\right)-\tau\left(a_{j}\right)\right|$ is less than the maximum. Assign weights to lines from the minimum weight of the sequence of $\gamma\left(s_{1}\right)<\gamma\left(s_{2}\right) \cdots<\gamma\left(s_{p}\right)$ and to points between corresponding points $a_{i}$ and $a_{j}$ then $F R_{L L}^{-1}$ is satisfied.

Case (iii): when $\left|\tau\left(a_{i}\right)-\tau\left(a_{j}\right)\right|<\frac{6 m-5}{10^{k}}$ and there is only one line between the points $a_{i}$ and $a_{j}$ i.e., $L=\left|n P_{s}\right|=1$,then the reciprocal of $\left|\tau\left(a_{i}\right)-\tau\left(a_{j}\right)\right|$ is more or as minimum as possible and the multiple of L with the reciprocal of $\left|\tau\left(a_{i}\right)-\tau\left(a_{j}\right)\right|$ is close to $\operatorname{diam}\left(F \operatorname{VPH}(m, n)<\left|n P_{b}\right|\right.$ by lemma 5.1.2, then it satisfies.

Case (iv) when $\left|\tau\left(a_{i}\right)-\tau\left(a_{j}\right)\right|>\frac{6 m-5}{10^{k}}$ and there are more than one line between the points $a_{i}$ and $a_{j}$ i.e., $L=\left|n P_{b}\right|>2$, then the reciprocal of $\left|\tau\left(a_{i}\right)-\tau\left(a_{j}\right)\right|$ multiplied by L is enough to reach .n- dimensional chemical graph satisfies, 
When $\mathrm{m}<\mathrm{n}$ the following cases hold true, Assign weight sof points column-wise

Case (i): When $\left|\tau\left(a_{i}\right)-\tau\left(a_{j}\right)\right|=\frac{i}{10^{k}}, \mathrm{i}=1,2$ and there is only one line between the points $a_{i}$ and $a_{j}$ i.e., $L=\left|n P_{b}\right|=1$, then the reciprocal of $\left|\tau\left(a_{i}\right)-\tau\left(a_{j}\right)\right|$ is more or less the maximum and satisfied.

Case (ii): Assign weights of points row wise $\left(C_{11}, C_{21} \ldots C_{m 1}, C_{m 2}, \ldots C_{m n}\right)$.

When $\left|\tau\left(a_{i}\right)-\tau\left(a_{j}\right)\right|=\frac{6 n-1}{10^{k}}, \mathrm{n}=2,3, \ldots$ and there is only one line between the points $a_{i}$ and $a_{j}$ (i.e.) $L=\left|n P_{b}\right|=1$, then the reciprocal of $\left|\tau\left(a_{i}\right)-\tau\left(a_{j}\right)\right|$ is less than the maximum. Assign weights to lines from the minimum weight of the sequence of $\gamma\left(s_{1}\right)<\gamma\left(s_{2}\right) \cdots<\gamma\left(s_{n}\right)$ and to points between corresponding points $a_{i}$ and $a_{j}$ then is satisfied.

Case (iii): when $\left|\tau\left(a_{i}\right)-\tau\left(a_{j}\right)\right|<\frac{6 n-1}{10^{k}}$, and there is only one line between the points $a_{i}$ and $a_{j}$ (i,e.) $L=\left|n P_{s}\right|=1$, then the reciprocal of $\left|\tau\left(a_{i}\right)-\tau\left(a_{j}\right)\right|$ is more or less the minimum, then the multiple of $\mathrm{L}$ with $\left|\tau\left(a_{i}\right)-\tau\left(a_{j}\right)\right|$ is close to $\operatorname{diam}(F V P H(m, n))<\left|n P_{b}\right|$ by lemma 5.1.2, thus it satisfies .

Case (iv): when $\left|\tau\left(a_{i}\right)-\tau\left(a_{j}\right)\right|>\frac{6 n-1}{10^{k}}$ and there are more than one line between the points $a_{i}$ and $a_{j}(\mathrm{i}, \mathrm{e}) L=\left|n P_{b}\right|>2$, then the reciprocal of $\left|\tau\left(a_{i}\right)-\tau\left(a_{j}\right)\right|$ multiplied by $\mathrm{L}$ is enough to reach $F R_{L L}^{-1}$. From the above cases, $\mathrm{n}$ - dimensional chemical graph satisfies,

Algorithm 5.1(a). Fuzzy radio reciprocal L- labeling number on Fuzzy V- Phenylenic Nano sheet FVPH (m, n ).

Step1: Assign weights to lines by $\gamma\left(s_{p}\right)=\left(\frac{\left(T \times 10^{q}\right)}{10^{k}}+\frac{p}{10^{k}}\right), R>T, T=5,6,7,8,9 ; p=1,2,3, \ldots$

If $R>T$ then go to step 1 and rearrange weights of $\tau\left(a_{t}\right)$ and $\gamma\left(s_{p}\right)$.

Step 2: Assign weights to points by $\tau\left(a_{t}\right)=\left(\frac{\left(R \times 10^{q}\right)}{10^{k}}+\frac{t}{10^{k}}\right), R=4,5,6,7,8,9 ; q=1,2,3, \ldots$ and $t=1,2,3, \ldots$

If $\mathrm{m} \geq \mathrm{n}$, assign weights to points row-wise

If $\mathrm{m}<\mathrm{n}$, assign weights to points column-wise.

Step 3: Find $\mu$ - distance for every pair of points $\delta\left(a_{i}, a_{j}\right), \mathrm{i}=1,2,3, \ldots, \mathrm{n}$ and $\mathrm{j}=1,2,3, \ldots, \mathrm{n}$;

$(i \neq j)$ by step 2

Step 4: Find Diam(FVPH $(m, n))$ using Step 3. If Diam $(F V P H(m, n))$ then go to step 5.

If $\operatorname{Diam}(\mathrm{FVPH}(\mathrm{m}, \mathrm{n}))$ then go to step 1 and rearrange weights of $\tau\left(a_{i}\right)$ and $\gamma\left(s_{i}\right)$.

Step 5: The highest of weight of the points is $F R_{L L}^{-1}(\mathrm{~m})$.

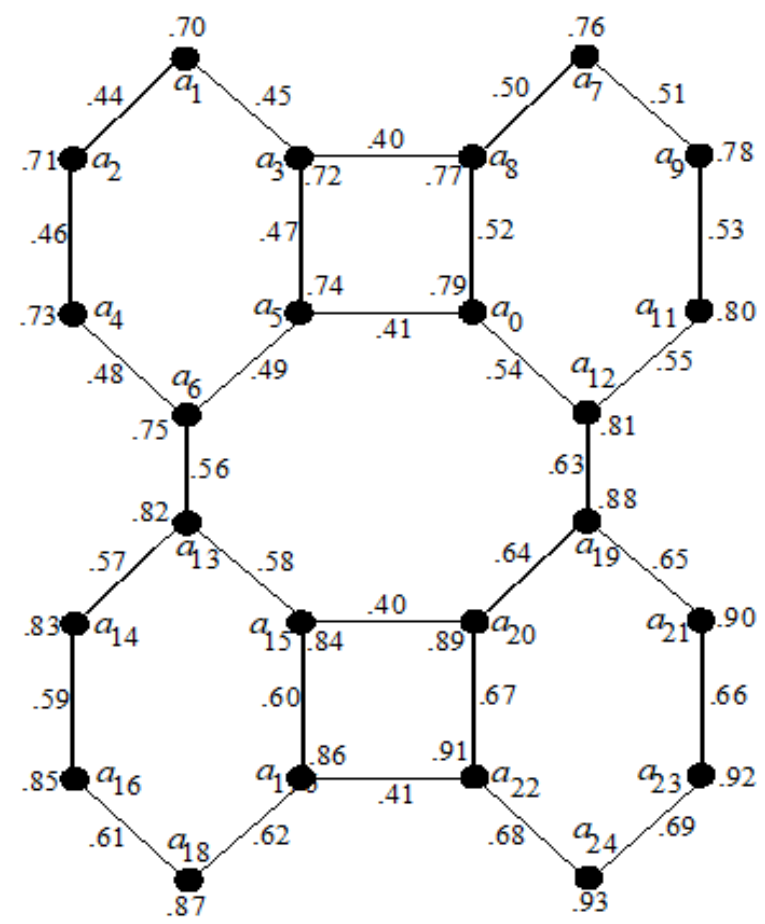

Fig 3. Fuzzy radio reciprocal L- labeling number is 0.93 


\subsection{Fuzzy radio reciprocal L- labeling on Sirpinski gasket graph $\left(\mathrm{S}_{n}\right)$}

Sirpinski gasket graph $\left(\mathrm{S}_{n}\right)$ is called Fuzzy Sirpinski gasket graph $F S_{n}=(A, \gamma, \tau)$ if it is a nonempty set. A together with a pair of functions $\tau: A \rightarrow[0,1]$ and $\gamma: S \rightarrow[0,1]$ with $\gamma\left(a_{i}, a_{j}\right)=\tau\left(a_{i}\right) \wedge \tau\left(a_{j}\right)$ for all $a_{i}, a_{j} \in A$. It is denoted by $F S_{n}$.

Theorem 5.2.1 : $F R_{L L}^{-1}(m)\left(F S_{n}\right)=\left(\frac{R}{10^{k}}+\frac{(A \mid}{10^{k+1}}\right)$.

Proof: Let $S_{n}$ be the n-dimensional Sirpinski gasket graph with points $A=\left\{a_{1}, a_{2}, a_{3} \ldots a_{n}\right\}$ and lines $S=\left\{s_{1}, s_{2}, s_{3} \ldots s_{n}\right\}$. Assign weights to points and lines

$$
\begin{aligned}
& \tau\left(a_{t}\right)=\left(\frac{\left(R \times 10^{q}\right)}{10^{k}}+\frac{t}{10^{k}}\right), R=5,6,7,8,9: q=1,2,3 \ldots ; t=0,1,2,3 \ldots \text { and } \\
& \gamma\left(s_{p}\right)=\left(\frac{\left(T \times 10^{q}\right)}{10^{k}}+\frac{p}{10^{k}}\right), R>T, T=5,6,7,8,9 \text { and } p=0,1,2,3 \ldots
\end{aligned}
$$

When $\mathrm{l}=1$, the $\delta\left(a_{i}, a_{i}\right)$ is always $1 \leq \delta\left(a_{i}, a_{j}\right)<2$, which gives $\operatorname{diam}\left(s_{n}\right)=\left|n P_{s}\right|$ by lemma 5.1.2. Assign weights to points from the top of $\left(s_{n-1}, T\right)$. Proceeding in a similar way assign weights from the top to bottom of $\left(\left(\mathrm{S}_{n-1}, \mathrm{~T},\left(\mathrm{~S}_{n-1}, \mathrm{~L}\right),\left(\mathrm{S}_{n-1}, \mathrm{R}\right)\right)\right.$ which gives the minimum possible difference of $\left|\tau\left(a_{i}\right)-\tau\left(a_{j}\right)\right|$ to reach $F R_{L L}^{-1}$. Therefore by $\tau\left(a_{t}\right)$ and $\gamma\left(s_{p}\right)$ the maximum weight of the point of is $\left(\left(\mathrm{S}_{n-1}, \mathrm{~T},\left(\mathrm{~S}_{n-1}, \mathrm{~L}\right),\left(\mathrm{S}_{n-1}, \mathrm{R}\right)\right)\left(\frac{R}{10^{k}}+\frac{(A \mid}{10^{k+1}}\right)\right.$.

Algorithm 5.2(a). Fuzzy radio reciprocal L- labeling number on Fuzzy Sirpinski gasket graph $\left(S_{\mathbf{n}}\right)$.

Step 1: Assign weights to points from $\left(\mathrm{S}_{n-1}, \mathrm{~T}\right)$ to $\left(\mathrm{S}_{n-1},\right)\left(\mathrm{S}_{n-1}, \mathrm{R}\right)$ by

$\tau\left(a_{t}\right)=\left(\frac{\left(R \times 10^{q}\right)}{10^{k}}+\frac{t}{10^{k+1}}\right), R=5,6,7,8,9: q=1,2,3 \ldots$ and $t=1,2,3 \ldots$

Step 2: Assign weights to lines from $\left(\mathrm{S}_{n-1}, \mathrm{~T}\right)$ to $\left(\mathrm{S}_{n-1}, \mathrm{~L}\right)\left(\mathrm{S}_{n-1}, \mathrm{R}\right)$ by

$\gamma\left(s_{p}\right)=\left(\frac{\left(T \times 10^{q}\right)}{10^{k}}+\frac{p}{10^{k+1}}\right), R>T, T=5,6,7,8,9 . p=1,2,3 \ldots$

If $R>T$ then go to step 1 and rearrange weights of $\tau\left(a_{i}\right)$ and $\gamma\left(s_{i}\right)$.

Step 3: Find $\mu$ - distance for every pair of points $\delta\left(a_{i}, a_{j}\right), \mathrm{i}=1,2,3, \ldots \mathrm{n}$ and $\mathrm{j}=1,2,3, \ldots \mathrm{n}(i \neq j)$

by step 2

Step 4: Find $\operatorname{diam}\left(S_{n}\right)$ using Step 3. If $\operatorname{diam}\left(S_{n}\right)<\left|n P_{c}\right|$, then go to step 5 .

If $\operatorname{diam}\left(\mathrm{S}_{n}\right)>\left|n P_{c}\right|$, then go to step 1 and rearrange the weights of $\tau\left(a_{i}\right)$ and $\gamma\left(s_{i}\right)$.

Step 5: The highest of weights of the points is $F R_{L L}^{-1}(m)$.

\subsection{Fuzzy radio reciprocal L- labeling on Sirpinski like graph $(S(n, 4))$}

Sirpinski Like graph $(\mathrm{S}(\mathrm{n}, 4))$ is called Fuzzy Sirpinski graph $F S(n, 4)=(A, \gamma, \tau)$ if a nonempty set together with a pair of functions $\tau: A \rightarrow[0,1]$ and $\gamma: S \rightarrow[0,1]$ with $\gamma\left(a_{i}, a_{j}\right) \leq \tau\left(a_{i}\right) \wedge \tau\left(a_{j}\right)$ for all $a_{i}, a_{j} \in A$. It is denoted by $F S(n, k)$.

Theorem 5.3.1: The fuzzy radio reciprocal L-labeling number of $S(n, 4)$ is the highest weight of the point of $S(n, 4)$.

Proof: Let $\mathrm{S}(\mathrm{n}, 4)$ be the $\mathrm{n}$-dimensional Nano sheet with points $A=\left\{a_{1}, a_{2}, a_{3} \ldots a_{n}\right\}$ and lines $S=\left\{s_{1}, s_{2}, s_{3} \ldots s_{n}\right\}$. Assign weights to points by

$\tau\left(a_{t}\right)=\left(\frac{\left(R \times 10^{q}\right)}{10^{k}}+\frac{t}{10^{k+1}}\right), \mathrm{t}=0,1,2,3, \ldots$

Proceeding in a similar way assign weights to lines by

$\gamma\left(s_{p}\right)=\left(\frac{\left(T \times 10^{q}\right)}{10^{k}}+\frac{p}{10^{k+1}}\right), R>T, \mathrm{p}=0,1,2,3, \ldots$

From above equations, $\gamma\left(s_{1}\right)<\gamma\left(s_{2}\right) \ldots<\gamma\left(s_{p}\right)<\tau\left(a_{1}\right)<\tau\left(a_{2}\right) \ldots<\tau\left(a_{t}\right)$

$\gamma\left(a_{i}, a_{j}\right)<\min \left(\tau\left(a_{1}\right), \tau\left(a_{1}\right)\right)$

Case(i): If $\mathrm{L}=1$ and $\left|\tau\left(a_{i}\right)-\tau\left(a_{j}\right)\right|=\frac{t}{10^{k}}, \mathrm{t}=1,2$ then the reciprocal of $\left|\tau\left(a_{i}\right)-\tau\left(a_{j}\right)\right|$ is more or less the maximum and it satisfies .

Case(ii): If $\mathrm{L}=1$ then $\left|\tau\left(a_{i}\right)-\tau\left(a_{j}\right)\right|$ is as follows,

For $n=1,\left(a_{1}, b_{1}, c_{1}\right)$

$n=2,\left(a_{1}, b_{1}, c_{1}\right),\left(a_{2}, b_{2}, c_{2}\right)$

$n=3,\left(a_{1}, b_{1}, c_{1}\right),\left(a_{2}, b_{2}, c_{2}\right),\left(a_{3}, b_{3}, c_{3}\right)$

Proceeding in this way $n=i,\left(a_{1}, b_{1}, c_{1}\right),\left(a_{2}, b_{2}, c_{2}\right),\left(a_{3}, b_{3}, c_{3}\right), \ldots,\left(a_{i}, b_{i}, c_{i}\right)$

Let $\left(f_{i}, g_{i}, h_{i}\right)$ be an ordered triplet which is $\left|\tau\left(a_{i}\right)-\tau\left(a_{j}\right)\right|$.

In this case $\left|\tau\left(a_{i}\right)-\tau\left(a_{j}\right)\right|$ will be

When $n=1,\left(f_{i}, g_{i}, h_{i}\right)$

$n=2,\left(f_{i}, g_{i}, h_{i}\right),\left(f_{i}, g_{i}, h_{i}\right)$

Similarly for $\mathrm{n}-$ dimensional $\mathrm{S}(\mathrm{n}, 4), \mathrm{n}$ times of $\left(f_{i}, g_{i}, h_{i}\right)$, where $f_{i}=\frac{3}{10^{k}}, g_{i}=\frac{2 f_{i}}{10^{k}}, h_{i}=\frac{3 f_{i}}{10^{k}}$. 
By lemma 5.1.2 $\operatorname{diam}(\mathrm{S}(\mathrm{n}, 4))<\left|n P_{s}\right|$. Hence $F R_{L L}^{-1}$ is satisfied.

Case(iii) If $L>2$ and the reciprocal of $\left|\tau\left(a_{i}\right)-\tau\left(a_{j}\right)\right|$ multiplied by $L$ is always greater than $\operatorname{diam}(\mathrm{S}(\mathrm{n}, 4))$, then it satisfies $F R_{L L}^{-1}$.

By (case i) and (case ii) it satisfies $F R_{L L}^{-1} \cdot|A|$ is $S(n, 4)$ point is the highest weight of $F S(n, 4)$.

Algorithm 5.3(a). Fuzzy radio reciprocal L- labeling number on Fuzzy Sirpinski like graph $(S(n, 4))$.

Step 1: Assign weights to points clockwise in every copy of $n-1$ by

$\tau\left(a_{t}\right)=\left(\frac{\left(R \times 10^{q}\right)}{10^{k}}+\frac{t}{10^{k+1}}\right), R=5,6,7,8,9 ; q=1,2,3 \ldots$ and $t=0,1,2,3 \ldots$

Step 2: Assign weights to lines clockwise in every copy of $n-1$, followed by the lines on the boundary and the remaining lines excluding the lines in the copies of $n-1$ by $\gamma\left(s_{p}\right)=\left(\frac{\left(T \times 10^{q}\right)}{10^{k}}+\frac{p}{10^{k+1}}\right), R>T, T=5,6,7,8,9 ; q=1,2,3 \ldots$ and $p=0,1,2,3 \ldots$

If $R>T$ then go to step 1 and rearrange weights of $\tau\left(a_{t}\right)$ and $\gamma\left(s_{p}\right)$.

Step 3: Find $\mu$ - distance for every pair of points $\delta\left(a_{i}, a_{j}\right), \mathrm{i}=1,2,3, \ldots \mathrm{n}$ and $\mathrm{j}=1,2,3, \ldots \mathrm{n}(i \neq j)$ by step 2

Step 4: Find $\operatorname{diam}(\mathrm{S}(\mathrm{n}, 4))$ by using Step 3. If $\operatorname{diam}(\mathrm{S}(\mathrm{n}, 4))<\left|n P_{c}\right|$, then go to step 5.

If $\operatorname{diam}(\mathrm{S}(\mathrm{n}, 4))$, then go to step 1 and rearrange weights of $\tau\left(a_{i}\right)$ and $\gamma\left(s_{i}\right)$.

Step 5: The highest of weight of the points is $F R_{L L}^{-1}(m)$.

\section{Application}

There are many routers with minimum dimensions and minimum distance which make more interference between any Wi-Fi routers. In this model of chemical graph, Wi-Fi routers are very close with minimum circumference area. Some of the routers have very minimum distance and maximum difference connected by one line. Assign frequencies to the routers by fuzzy weights from the interval 0 and 1 . If the weight of every router lies between $(0.5 \times x x \ldots x \mathrm{GHz}, 0.9 \mathrm{xxx} \ldots \mathrm{x} \mathrm{GHz})$ then the signal is very strong. WD- Wi-Fi router capacity difference, DS- Distance between Routers, L- Number of lines in between routers, I- Interference, SS- Signal Strength. Here the of WD and DS are given by assigning two decimals of fuzzy weights. Tables values of WD and DS varies depending on their decimals weights.

Let A, B, C, D, E, F are six Wi-Fi routers connected by line. Assign weights to lines and points from (0.5xxx, 0.9xxx). Here consider weights of points as Wi-Fi router frequencies and the minimum $\mu$ - length of any two Wi-Fi routers is consider as a distance (i.e.) kilometer(KM).

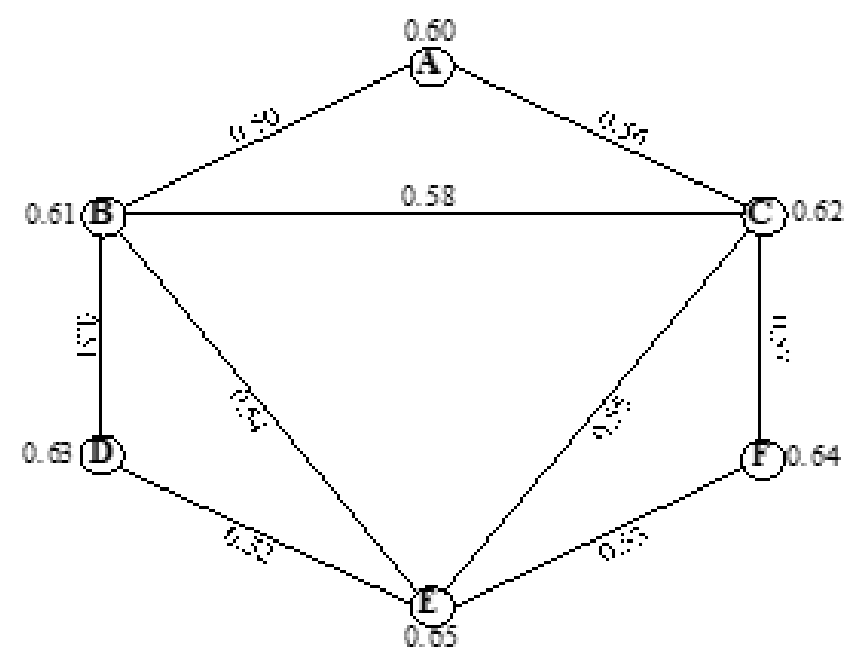

Fig 4. Fuzzy chemical graph

From Figure 4 , Find $\mu$ - distance:

$\delta(A, B)=2 \mathrm{~km}, \delta(A, C)=3.96 \mathrm{~km}, \delta(A, D)=1.78 \mathrm{~km}, \delta(A, E)=3.79 \mathrm{~km}, \delta(A, F)=3.53 \mathrm{~km}, \delta(B, A)=2 \mathrm{~km}$, $\delta(B, C)=1.72 \mathrm{~km}, \delta(B, D)=1.96 \mathrm{~km}, \delta(B, E)=1.85 \mathrm{~km}, \delta(B, F)=3.47 \mathrm{~km}, \delta(C, A)=1.78 \mathrm{~km}, \delta(C, B)=1.72 \mathrm{~km}$, $\delta(C, D)=3.68 \mathrm{~km}, \delta(C, E)=1.81 \mathrm{~km}, \delta(C, F)=1.75 \mathrm{~km}, \delta(D, A)=3.96 \mathrm{~km}, \delta(D, B)=1.96 \mathrm{~km}, \delta(D, C)=3.68 \mathrm{~km}$, $\delta(D, E)=1.92 \mathrm{~km}, \delta(D, F)=3.80 \mathrm{~km}, \delta(E, A)=3.79 \mathrm{~km}, \delta(E, B)=1.85 \mathrm{~km}, \delta(E, C)=1.81 \mathrm{~km}, \delta(E, D)=1.92 \mathrm{~km}$, 
$\delta(D, F)=1.88 \mathrm{~km}, \delta(F, A)=3.53 \mathrm{~km}, \delta(F, B)=3.47 \mathrm{~km}, \delta(F, C)=1.75 \mathrm{~km}, \delta(F, D)=3.80 \mathrm{~km}, \delta(F, E)=1.88 \mathrm{~km}$. Diameter of fuzzy graph is $3.96 \mathrm{~km}$.

Table 1. Fuzzy radio reciprocal L -labeling.

\begin{tabular}{llll}
\hline $\mathrm{x}=\mathrm{WD}(\mathrm{GHz})$ & $\mathrm{d}=\mathrm{DS}(\mathrm{KM})$ & $\mathrm{L}$ & $\mathrm{D}=3.96$ \\
\hline $0.01 \mathrm{GHz} \leq \mathrm{x} \leq 0.04 \mathrm{GHz}$ & $2.00 \mathrm{~km}, 1.96 \mathrm{~km}, 1.92 \mathrm{~km}, 1.88 \mathrm{~km}, 1.85 \mathrm{~km}, 1.81 \mathrm{~km}, 1.78 \mathrm{~km}$, & 1 & $\mathrm{~d}+1 \times \frac{1}{x}>3.96$ \\
& $1.75 \mathrm{~km}, 1.72 \mathrm{~km}$. & \\
\hline $0.01 \mathrm{GHz} \leq \mathrm{x} \leq 0.05 \mathrm{GHz}$ & $3.96 \mathrm{~km}, 3.80 \mathrm{~km}, 3.79 \mathrm{~km}, 3.68 \mathrm{~km}, 3.53 \mathrm{~km}, 3.47 \mathrm{~km}$ & 2 & $\mathrm{~d}+2 \times \frac{1}{x}>3.96$ \\
\hline
\end{tabular}

If $\mathrm{Wi}-\mathrm{Fi}$ routers are assigned based on fuzzy weights of figure 5.1, then there is no interference in between the routers and the strength of signals are good or excellent.

\section{Conclusion}

In this study, the methodology was introduced for fuzzy radio reciprocal L-labeling. Here the general formula of fuzzy radio reciprocal L-labeling has been mathematically derived to apply this concept in chemical graphs with further result and discussions. In this connection the frequencies of $\mathrm{Wi}-\mathrm{Fi}$ are assigned to the structure of chemical graphs. The generalized formula applied to certain chemicals graphs are N-tube, Sirpinski gasket graph and Sirpinski like graph satisfy fuzzy radio reciprocal L-labeling where every frequency of channels is from the closed interval 0 to 1.

\section{References}

1) Hale WK. Frequency assignment: Theory and application. In: Proceeding of the IEEE;vol. 68. 1980;p. 1497-1514.

2) R J. Griggs Labeling Graphs with a Condition at Distance 2. Siam Journal on Discrete Mathematics. 1992;5(4):586-595.

3) Heuvel JVD, Leese RA, Shepherd MA. Shepherd Graph labeling and radio channel assignment. Journal of Graph Theory. 1998;29(4):263-283. Available from: https://dl.acm.org/doi/10.5555/1379171.1379176.

4) Shao Z, Zhang D. The L(2,1)- labeling on graphs and the frequency assignment problem. Applied Mathematics Letters. 2008;21(1):37-41. Available from: https://doi.org/10.1016/j.aml.2006.08.029.

5) Gani AN, Radha K. The Degree of a Vertex in some Fuzzy Graphs. International Journal of Algorithms, Computing and Mathematics, Eashwar Publications. 2009;3(2):107-116.

6) Panigrahi AP. Survey on Radio k-Colorings of Graphs. AKCE International Journal of Graphs and Combinatorics. 2009;6(1):161-169. Available from: 10.1080/09728600.2009.12088883.

7) Massa'deh MO, Gharaibeh KN. Some Properties on Fuzzy Graphs. Advances in Fuzzy Mathematics. Research India Publications. 2011;2(6):245-252. Available from: http://www.ripublication.com/afm.htm.

8) Gani AN, Subahashini R. A Note on Fuzzy Labeling. International Journal of Fuzzy Mathematical Archive. 2014;4(2):88-95.

9) Nagoorgani A, Subahashini DR. Fuzzy Labeling Tree. International Journal of Pure and Apllied Mathematics. 2014;90(2):131-141. Available from: https://dx.doi.org/10.12732/ijpam.v90i2.3.

10) Tom M, Sunith MS. Muraleedhtan Shetty sunith. Strong sum distance in fuzzy graph. Springar Plus. 2015;4(214). Available from: https://doi.org/10.1186/ s40064-015-0935-5.

11) Nazeer S, Khan S, Kausar I, Nazeer W. Radio Labelling and Radio Number for Generalized Caterpillar Graphs. Journal of Applied Mathematics and Informatics. 2016;34(6):551-565. Available from: 10.14317/jami.2016.451.

12) Mahapatra R, Samanta S, Allahviranloo T, Pal M. Madhumangal Pal. Radio fuzzy graphs and assignment of frequency in radio stations. Computational and Applied Mathematics. 2019;38(4):1-2. Available from: https://link.springer.com/article/10.1007/s40314-019-0888-3.

13) John BS, Mela JV. Radio labeling of complete related graphs. Compliance Engineering Journal. 2020;11(2):173-187.

14) Uma J, Bhargavi RM. Radio labeling on some corona graphs. AIP Conference Proceeding. 2020;2227:1-7. Available from: https://doi.org/10.1063/5. 0025217.

15) Chavez A, Liu DDF, Shurman M. Optimal radio-k-labelings of trees. European Journal of Combinatorics. 2021;91(91):103203. Available from: https://dx.doi.org/10.1016/j.ejc.2020.103203.

16) Chartrand G, Erwin D, Zhang P. A graph labelling problem suggested by FM channel restrictions. Bull Inst Combin Appl. 2005;43(114):43-57.

17) Mordeson JN, Nair PS. Series Title Studies in Fuzziness and Soft Computing. In: Fuzzy Graphs and Fuzzy Hypergraphs. 2000;p. 19-81. Available from: https://doi.org/10.1007/978-3-7908-1854-3.

18) Sunitha MS, Mathew S. Fuzzy Graph Theory: A Survey. Annals of Pure and Applied Mathematics. 2013;4(1):92-110.

19) Gani AN, Akram M, (a) Subahashini DR. Novel Properties of Fuzzy Labeling Graphs. Journal of Mathematics. 2014;2014(70):1-6. Available from: https://dx.doi.org/10.1155/2014/375135.

20) Xavier A, Theresal SS, Raja MJ. Induced H-packing K-Partition number for certain nanotubes and chemical graphs. Journal of Mathematical Chemistry. 2020;(58):1177-1196. Available from: https://link.springer.com/article/10.1007/s10910-020-01124-3.

21) Xavier DA, Rosary M, Arokiaraj A. Topological Properties of Sierpinski Gasket Rhombus Graphs. International Journal of Mathematics and Soft Computing. 2014;4(2):95-95. Available from: https://dx.doi.org/10.26708/ijmsc.2014.2.4.10.

22) Vinoth SA, Bharathi T. Radio Reciprocal Membership Function on Cycle Related Graphs. International Journal of Recent Technology and Engineering. 2019;4(8). Available from: https://www.ijrte.org/wp-content/uploads/papers/v8i4/B3583078219.pdf. 